\title{
DESIGN OF DRESSING STATIONS AND CONTROL OF WOUND INFECTION
}

\author{
BY
}

\author{
WILliaM GISSANE, A. A. MILES and R. E. O. WILliAMS
}

From the Clinical Staff and Medical Research Council Unit, Birmingham Accident Hospital

\section{Introduction}

In spite of the recent advances in the treatment of wounds by antiseptic and chemotherapeutic agents, the aseptic treatment of wounds still remains the most effective way of controlling the infection that may occur during treatment. Aseptic technique is seen in greatest elaboration in the operating theatre, but theatre technique is obviously impracticable in wards, out-patient departments and factory surgeries, where asepsis is nevertheless of equal importance. Simple and practicable precautions can be devised for these places. However, recent surveys in wards and out-patient departments have revealed a high incidence of wound infection due to defects in the aseptic dressing of the wounds (see M.R.C. War Memo. No. 6). The defects are not altogether due to the difficulties of surgical practice or the greater frequency of dirty and damaged wound tissue in war-time; defective aseptic techniques can also be found in civilian practice where peace-time conditions prevail.

The appearance of gross and sometimes fatal sepsis can be traced in many instances to infection of the wound during its treatment. In industry these cases are referred to hospital, with the result that sepsis of industrial wounds looms large in hospital practice, but does not strike the factory staff as anything more than a rare occurrence. Many wounds are already septic when first seen in the factory surgery, but a proportion of open wounds become septic during treatment. It has been argued that this latter sepsis is not due to any gross breaches in aseptic precautions, but to accidents whose prevention could be achieved only by impracticable elaboration of the routine of treatment. The argument is, we think, invalid, because the incidence of sepsis can be reduced by simple revision of dressing technique. But even if it were valid, there would still be a strong case for a careful review of existing methods, for obvious sepsis is not the only result of cross-infection of wounds. Our recent bacteriological survey of wounds in factory surgeries and out-patient departments (unpublished) has shown that from $\mathbf{3 0}$ to $\mathbf{4 0}$ per cent. of all clinically clean wounds more than one day old are ' silently' infected, usually with Staph. aureus and sometimes with Str. pyogenes. The results of sepsis are well recognized: quite apart from frequent permanent disabilities, the sepsis is responsible for prolonged attendance at hospital and prolonged absence from work. Similarly, but to a lesser degree, silent infection prolongs the period of treatment of the wound. For example, the mean duration of hospital treatment of 188 minor sutured wounds of the hand, classified into clean, silently infected, and clinically septic, was as follows:

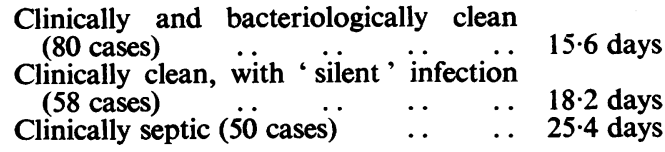

Taking these small wounds as an example, it is obvious that the time, both patient's and hospital's, that would be saved by eliminating silent infection of a wound is not great (about 18 per cent.). But the saving, even in patients with such wounds, becomes highly significant when we consider the incidence of silent infection and the total number of wounds which may be silently infected. The silent infection rate may be as high as $\mathbf{4 0}$ per cent.' and, as an example of the number of wounded persons treated, we may quote figures from a group of large factories employing 103,000 workers, where, in 1942, no less than 430,310 first dressings and 615,333 redressings were performed. (Dr. Donald Stewart, personal communication.)

Silent infections occur in all kinds of wounds and may have other consequences beside that of delayed healing. For example, in our view it is not unreasonable to attribute to it a common feature of many wounds said to have healed by first intention. These wounds, after a period of healing unaccompanied by sepsis, develop a scar which remains red and swollen, and which may for months be so tender as to constitute a prolonged disability. It is highly probable that this scar represents a reaction to silent infection; the point is under investigation.

There is another important aspect of silently infected wounds. They are particularly dangerous in a dressing station, whether of factory surgery or out-patient department, because they are unrecog- 
nized sources of large numbers of pathogenic bacteria. By definition, they cannot be recognized clinically, and are consequently treated as clean wounds. It is a matter of frequent observation that medical and nursing staff will treat a series of apparently clean wounds with no more than the most perfunctory aseptic and antiseptic precautions for each patient, and with none whatsoever to prevent the spread of hypothetical infected material from one patient to the next.

The rapid succession of patients with silently infected wounds in a busy dressing station means that a large reservoir of infection is constantly present. A good dressing technique must therefore minimize the incidence of clinical sepsis, which may be small, and of the silent infection, which constitutes, numerically at least, a bigger problem.

Unfortunately, the reduction of silent infection can be demonstrated only by a bacteriological study over a long period. But wherever we have made these studies we have found a high incidence of added silent infection, a fact which suggests that aseptic routines as generally practised could be greatly improved in this respect.

The possible sources from which a wound may become infected with Staphylococcus aureus and Streptococcus pyogenes are: the nose, throat and skin of the patient himself or of the nurses and doctors who attend to his wound, and, in the factory surgery and hospital particularly, infected wounds of other patients. The occurrence of wound to wound infection has been attested by several recent reports (Miles et al., 1940; Spooner, 1941; McKissock, et al. 1941) and McKissock et al. were able to show that it could be controlled by the introduction of a dressing technique designed to minimize the transference of pathogenic organisms from one wound to another. We have been able to confirm these observations (Williams, ClaytonCooper, Howat and Miles, unpublished).

We recognize that the maintenance of a completely sterile wound is an unattainable ideal. Infection depends both on the susceptibility of the tissues and the dosage of pathogenic bacteria introduced into them. The treatment of the wound helps to reduce its susceptibility to infection. Aseptic measures, by eliminating several of the more important sources of infection, reduce the infecting bacteria to numbers that the tissues can overcome.

\section{Routine of Wound Dressing}

The best general method of reducing added infection is by revision of the dressing technique. We use the word ' revision' rather than 'improvement' or 'elaboration' because, from observation of a large number of dressing stations, it is clear that though some preventive measures are inadequate, others (e.g. 'scrubbing-up' instead of the use of dry hands and a 'no-touch' technique) are unnecessarily elaborate. In many cases, the routine can with advantage be 'rationalized' and the time thereby saved is more than enough for taking additional aseptic precautions.

For the most part dressings are, from a bacteriological point of view, either carelessly or very badly done. A careful scrutiny of any routine will yield a list of preventable breaches in the anti-bacterial protection that it is supposed to afford. But to offer a list of remedies to a busy medical staff is, on the face of it, to offer an additional burden of work. The minutiae of the aseptic routine and the therapeutic manipulations are already reduced to a practicable minimum. The staff is not prepared to spend time on improvements whose value only a long-term application will reveal.

The problem, therefore, is to introduce a dressing routine which respects silent infection as well as obvious sepsis without increasing the time spent on each patient, and its solution lies in the analysis of existing routines from the standpoint of functional efficiency. Most dressing stations are no more than rooms furnished with a number of medical appliances-trolley, sink and perhaps sterilizerplaced without much regard to their function. If the wash-basin is 12 feet from the patient and dressing trolley, the wash-basin tends to drop out of use. If the sterilizer is relatively inaccessible, either the sterilization is omitted or it is done badly on the trolley with far too dilute antiseptic.

The main routes of added infection are: $(a)$ the hands and pocket scissors of the dressers: (b) the trolley instruments; and (c) 'clean' dressings, lotions and their containers, all of which may have been contaminated in bulk when portions are removed by contaminated. hands and instruments while a dressing is in progress. Carriage of infection on the hands can be prevented by washing after each dressing, and contamination on scissors by keeping them in antiseptic.

Contamination of clean dressings in bulk can be avoided in many ways. The single-handed dresser may use packets containing enough material for one wound, though this method is time-consuming for large numbers of small wounds. Alternatively, before each dressing the requisite amount of material can be gathered in a sterile bowl with sterile instruments from the clean store. This we term the 'cafeteria' method. The required aseptic conditions can also be achieved by the provision of containers for bulk dressing material that allow withdrawal under sterile conditions without lidlifting, etc.- ' safe-sampling' containers. The design of such a container is under consideration.

Two nurses may act as dresser and server. The server hands to the dresser everything required from clean stocks, and never comes into contact with infected material. She also looks after the trolley supplies for each dressing and supervises the sterilization of instruments, etc. One server can supply two or perhaps three dressers in a dressing station working to full capacity.

We will consider first the single-handed dresser, and later the modifications required for dressing teams of two or more. The ideal dressing routine 
for an open wound is briefly: setting of the dressing trolley; removal and safe discard of soiled dressings; inspection and toilet of the wound; dressing of the wound with clean material; cleaning and sterilization of instruments, bowls, etc., used in the dressing; washing of the hands; and, finally, recording the treatment of the patient. In practice, the routine is often: unbandaging and inspection of the wound; selection of material; toilet and dressing of the wound; recording of treatment; clearing up of soiled and unused dressings; and, finally, proceeding to the next patient.

The requirements for the ideal procedure are briefly as follows:

1. Trolley, with sterile or 'clean' (where 'clean' means 'free from pathogenic bacteria') dressing materials, preferably in safe sampling containers from which portions can be taken without contaminating the whole store.

2. A near-by deep and capacious discard bin.

3. Sink for washing instruments.

4. Sterilizer.

5. Wash-basin (may be the same as No. 3).

6. Records desk.

7. Cupboard or shelves for spare dressing stores, etc.

In factory surgeries a good deal of dirty material on wounds might be removed by the patient himself, and a separate patients' wash-basin and discard bin, to facilitate preliminary cleansing of the parts surrounding the wound, might be added to the list.

\section{Lay-out of the Dressing Station}

The awkwardness of the design of existing dressing stations, for the purposes we have outlined, is exemplified in Plans I-VI, which are based on some of the surgeries we have visited. They are selfexplanatory. The seven essential pieces of furniture are indicated, where they exist, by the numbers given in the above list of requirements. The order is that in which the different pieces of equipment would be used during a wound dressing properly carried out in the way outlined above. The bin, No. 2, will not be found in most of the diagrams; soiled dressings were usually discarded into a shallow bowl kept on the lower shelf of the trolley, of such capacity that it was filled to overflowing after half an hour's continuous work. In the plans the path followed by the nurse is shown by the continuous line with arrows, that followed by the patient by the broken line.

Surgery $I$ is shown twice. In Ia the path taken by the dresser during her routine is plotted; in Ib, the path she would have taken if following the ' ideal' routine. In II, III, IV, V and VI the paths are those necessary for carrying out the 'ideal' technique. The path in most instances includes a visit to the store cupboard. In practice, the paths usually lay between the dressing trolley and the records desk (1 and 6), and a glance at the plans of these surgeries shows that even for the practice of an inadequate dressing technique, the lay-out is not efficient. In the treatment of 40 patients in one morning, a nurse might walk at least an unnecessary half mile.

Plan VII represents a hypothetical small surgery ( 12 feet by 12 feet) where this movement is reduced to a minimum. The principles of its layout are:

1. The essential pieces of furniture are gathered around the dresser, in the best light, and away from the door. They are placed so that the dresser, when standing ready to deal with a patient, has to take a maximum of one or two paces to reach the trolley, the records desk or the wash-basin and sterilizer respectively.

2. Where movement about the surgery is essential the patient, rather than the dresser, does the walking. But the plan is so arranged that at no point does the track taken by the patient from door to waiting bench, to his wash-basin, to the dressing chair and out of the room again, cross that taken by the nurse in carrying out her duties as a dresser.

A second wash-basin is provided for patients. A third is placed near the couch, so that if dressings are to be done on a recumbent patient, a bacteriologically safe unit is constituted by moving the trolley over to the couch side. The line around the couch indicates the position of an overhead trolley rail from which depends a curtain that can be drawn at will around the couch.

Plans VIII and IX show respectively an outpatient dressing station and a ward side-room at the Birmingham Accident Hospital, which were devised by the application of the principles outlined above. Plan VIII was designed to accommodate a doctor and his secretary, four nurses (two acting as dressers and two as servers) and two patients at one time. Each member of the staff has ready access to his work and to a wash-basin. The servers work outside the low walls WW (5 feet high), passing clean material through one hatch, and taking soiled instruments, etc., through another. The dressers work from a fixed dressing bench. Dressings are discarded into a bin in an enclosed cupboard under dressing slab. The bin is removed for emptying through a door outside the dressing station. The doctor sees each patient with no more movement than the turning of a swivel chair. Should he handle a wound, the wash-basin is immediately behind him for his use. The paths taken by the dressers and by the patients are plotted in the plan.

Plan IX is of a side-room of a ward used mainly for patients with septic wounds of the hand, adapted to the needs of a four-nurse team dressing technique. The team consists of two dressers, a server and a runner. The dressers share one trolley. Here the function of the dressers is simpler than in a factory, since there are no records to be written during the dressings. This arrangement has been in use for 14 months, and, once the nurses had learnt their respective duties in the team, proved to be generally as well as bacteriologically efficient.

Factors Determining Design

The factors that must be considered in designing a dressing station comprise those which are common 


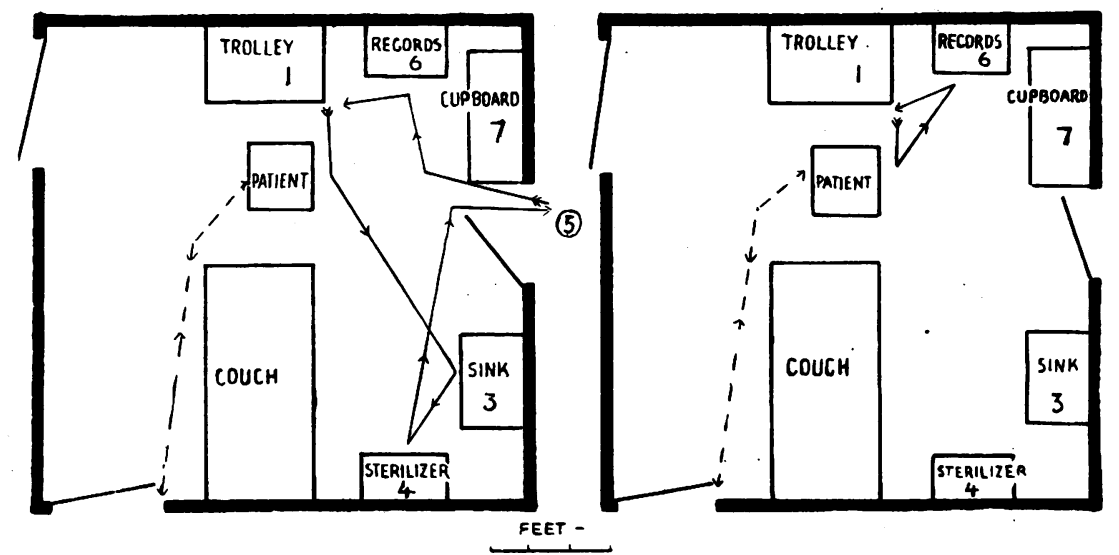

Plan Ia.

Plan Ib.

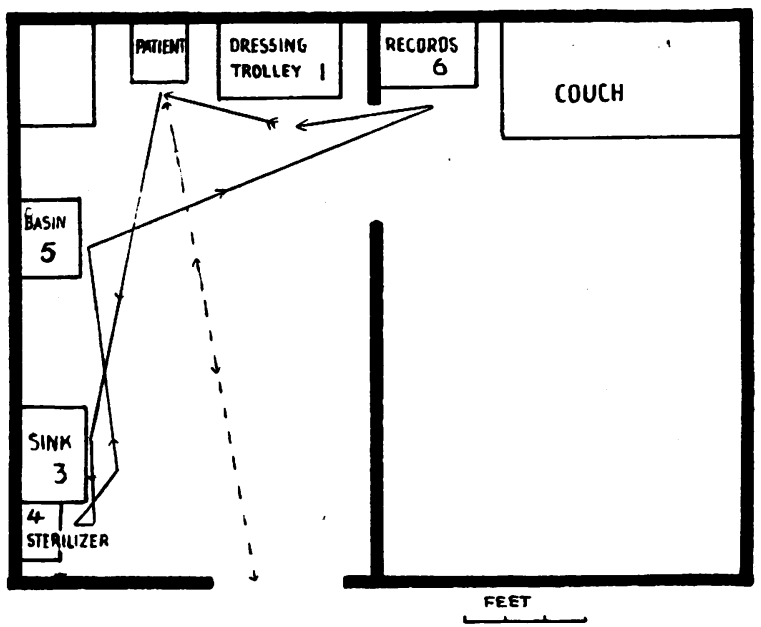

Plan II.

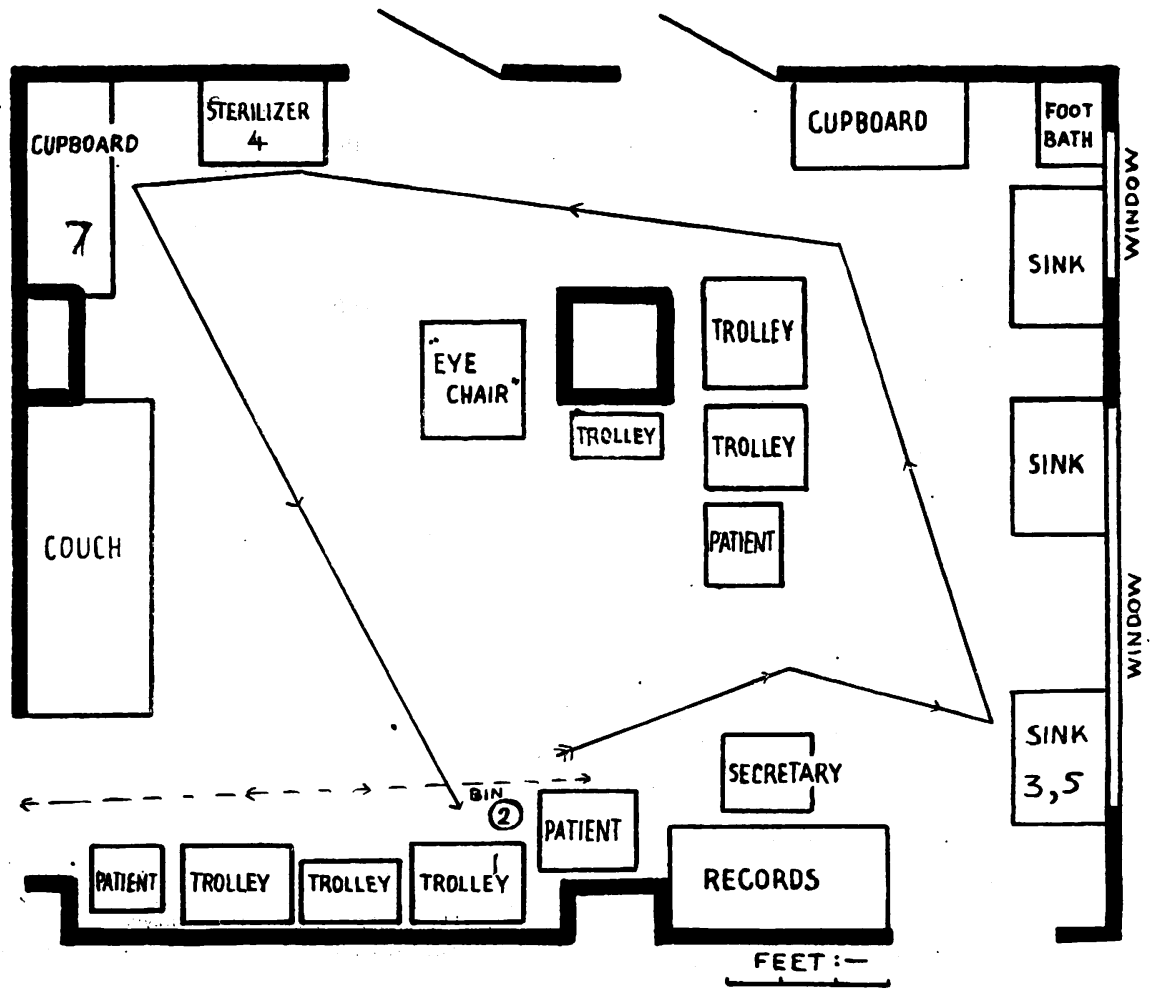

Plan III. 

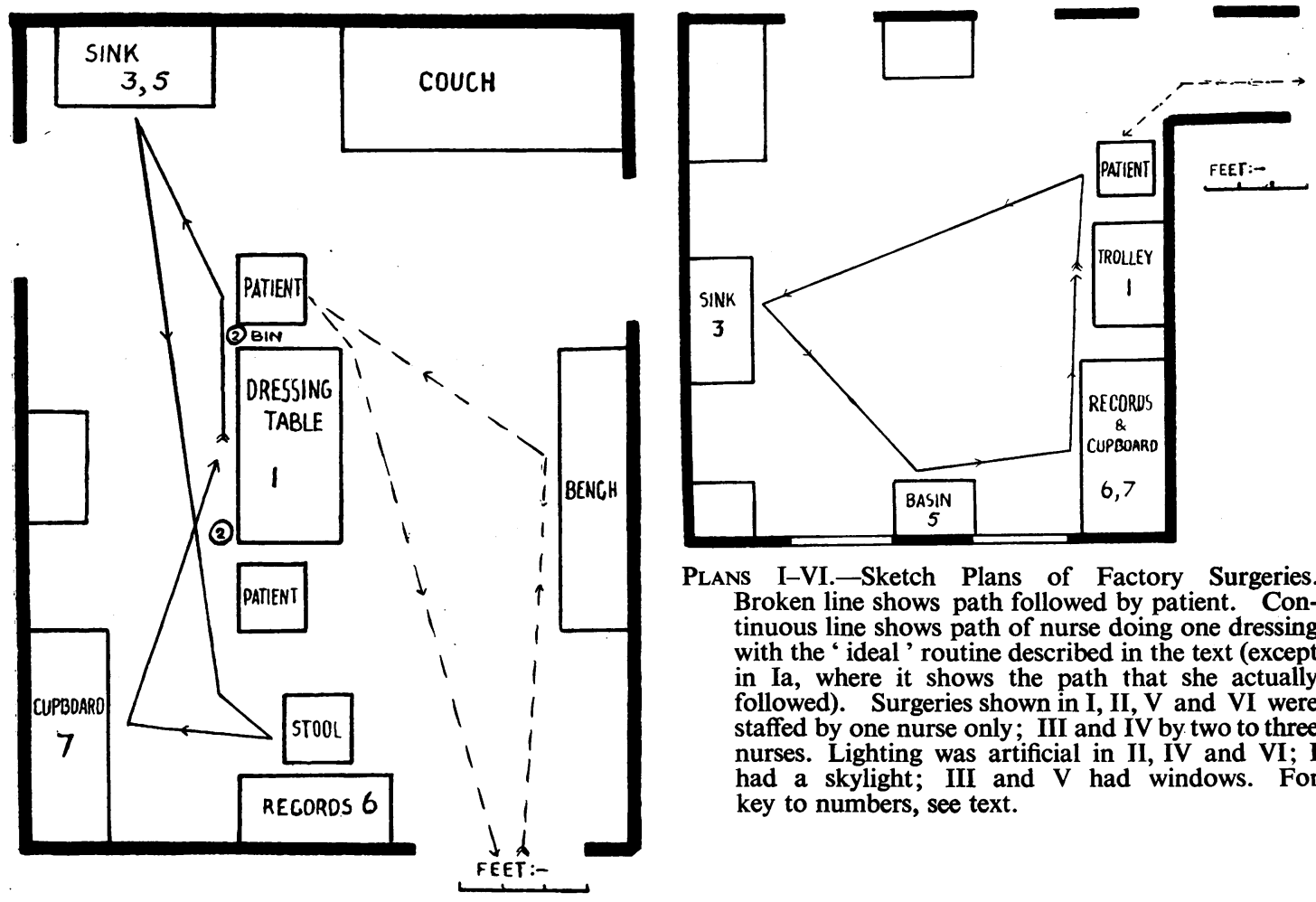

Plans I-VI.-Sketch Plans of Factory Surgeries. Broken line shows path followed by patient. Continuous line shows path of nurse doing one dressing with the 'ideal ' routine described in the text (except in Ia, where it shows the path that she actually followed). Surgeries shown in I, II, V and VI were staffed by one nurse only; III and IV by two to three nurses. Lighting was artificial in II, IV and VI; I had a skylight; III and V had windows. For key to numbers, see text.

Plan IV.
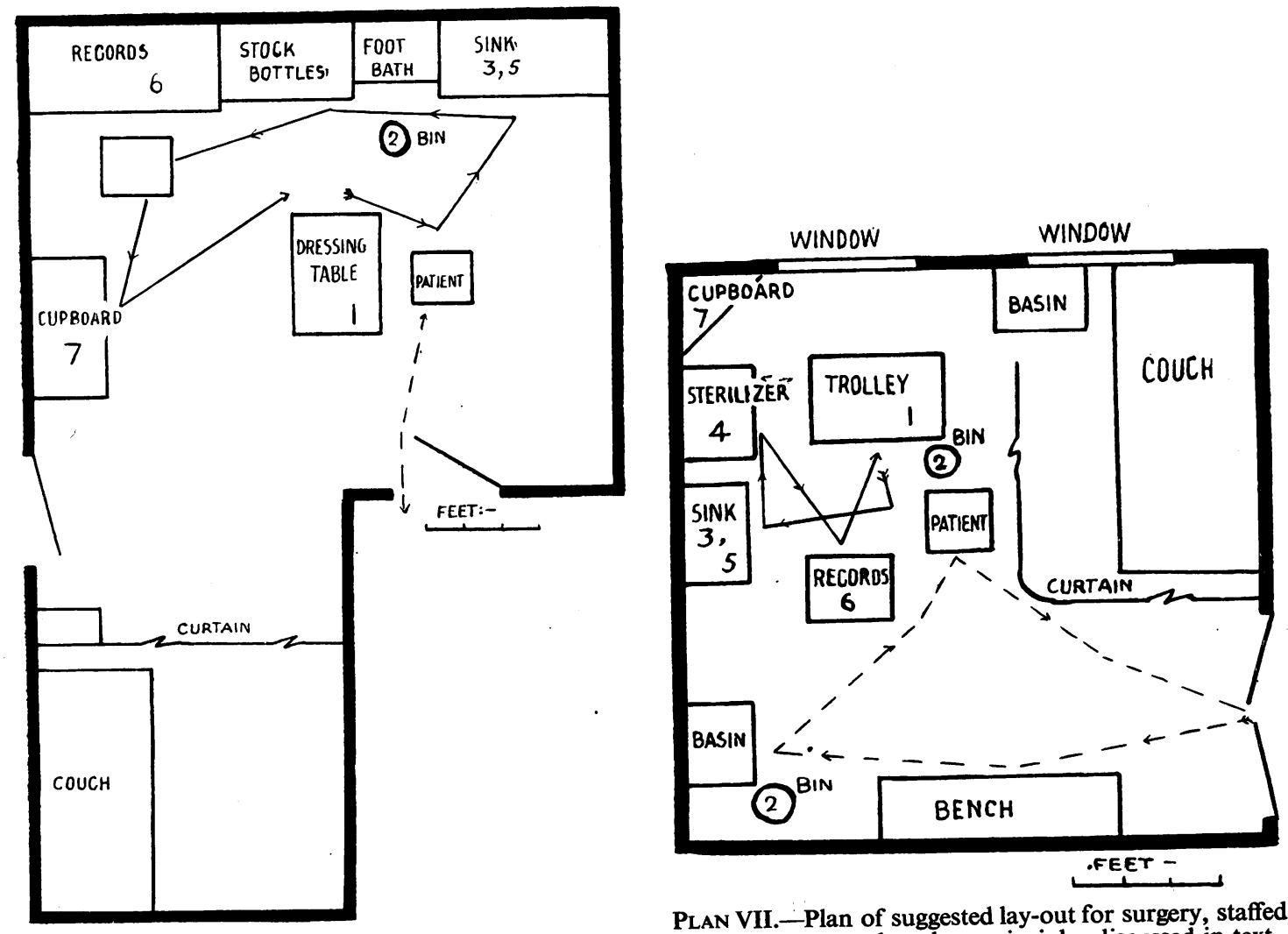

Plan V.

Plan VII.-Plan of suggested lay-out for surgery, staffed by one nurse, based on principles discussed in text. 


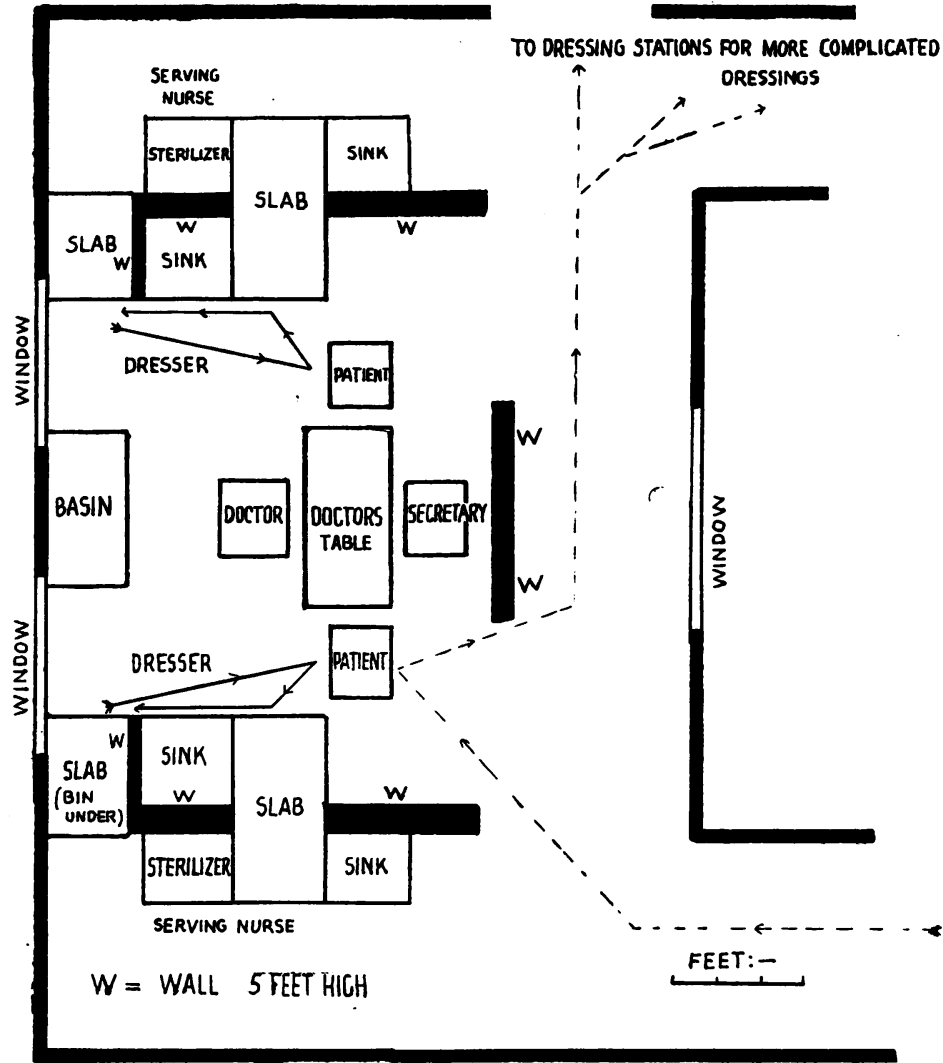

Plan VIII.-Part of the New Out-Patient Department, Birmingham Accident Hospital.

to works surgeries and to hospital departments, and those which are, for the most part, peculiar to works. The list that follows is not intended to be comprehensive in respect of works factors; we do not as yet know enough about them.

1. Geographical.-The dressing station must be readily accessible. The number of injuries per

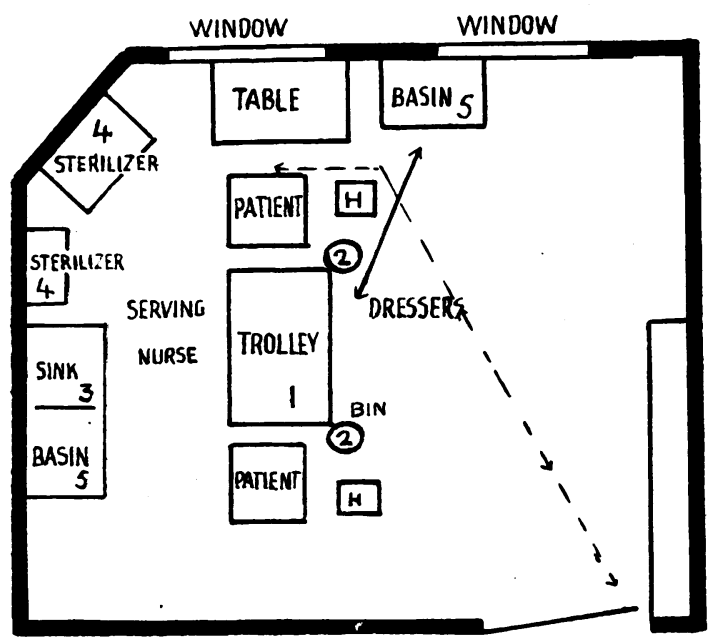

$H=$ HAND-REST

FEET:-

Plan IX.-Ward Dressing Station, Birmingham Accident Hospital, for two dressers working simultaneously. person per month reported in a works surgery that was not more than 50 yards from the remotest worker, was 4.5 times the number in a comparable room (in a factory doing similar work, under the supervision of the same medical officer) where some of the workers had to walk 10 minutes to get to it. (Dr. E. H. Capel, personal communication.) Stairs, and a walk in the open air, are also said to discourage attendance.

2. Structural.-The rooms should be spacious enough for the staff and work intended. Day lighting is preferable, but good artificial lighting, especially fluorescent tubular lighting, is satisfactory for minor surgical manipulations.

The position of the doors and windows in the room is usually fixed. The dressing area, where the essential furniture (1-6 in the list above) will be disposed, should be as remote as possible from the door, and placed so that the path taken by the ingoing and outgoing patients does not cross the path taken by the nurses. In larger dressing stations, designed for more than one dresser with servers, the design must ensure that the paths of the servers and dressers do not intersect more than is necessary. If the dressing area can be placed under a window, so much the better; but daylighting should be sacrificed to drainage. The sacrifice is often necessary, for water points and drains, being expensive to alter or extend; often define the position of the sinks and wash-basins, 
and it is part of our thesis that awkwardly placed basins frequently determine that omission of hand washing and instrument cleansing which constitutes the largest gap in the defence against surgical infection. In general, the wash-basin and sink should be close together, and in the middle of the wall along which the supply of drainage and water points is most convenient. From that fixed point the remainder of the dressing area may be planned. The table with the sterilizer adjoins the sink against the wall. A shallow stock cupboard hangs at face level above the basin and sink. The dresser will work with her back to the hand-basin and sink, with the dressing trolley, discard bin and records desk conveniently disposed at one side or the other. The patient's chair is immediately in front of the dresser. The records desk should be at standing height (a foot rest for leg injuries is conveniently stored under it). The records desk accommodates the day sheet, special sheets, certificates, etc. In larger surgeries there may be a single records desk or office, in charge of a clerk, and here it is more convenient to give each patient a small card upon which all his notes are written. In such a case only a small records desk is needed, or a small hinged flap may be added to the dressing trolley to accommodate the individual cards.

The trolley should be on wheels, so that it can be moved, if necessary, to a couch in the cubicle. The curtained cubicle, if it is far from the hand-basin, should be provided with a separate hand-basin. If possible, the curtains should run on suspended trolley rails, so that the floor is not encumbered with upright rail supports.

3. Social Considerations.-In many factories, especially those that in peace-time employed women, there is a strong tradition of segregation of the sexes in a surgery. In others, the practice is similar to that of hospital out-patient departments, where there is no segregation. The manipulations that require exposure of the covered parts are carried out in a cubicle. The cubicle method, however, is often inconvenient, for either the whole paraphernalia of trolley, etc., must be taken over to the cubicle, or the dressing done by an imperfect technique. It seems to us that the principle of a dependent curtain rail, suspended at a height of 6 feet 6 inches along the periphery of the dressing area nearest to the waiting patients, round which an easily running curtain might be drawn to seclude the seated patient deserves trial as a convenient and time-saving device. It has the advantage that in small surgeries, the cubicle, which usually contains the couch, is left free for patients suffering from faints, shock and other medical conditions needing relatively long treatment.

The provision of a basin at which the work-soiled patient can give himself a preliminary wash worked well in one factory surgery we inspected, but its use must be carefully supervised and individual towels must be provided; otherwise it might become a potent source of cross-infection of wounds.

4. Technical Considerations.-In the small surgery, where simplicity of procedure is essential to a rapid turnover of patients without too great a lag at the rush hours, several technical improvements on the usual procedure are possible. Among these we may note:

(a) The provision of readily sterilizable lidded bowls in which dry, sterile instruments and dressings may be stored.

(b) The provision of safe-sampling containers for sterile dressings, gauze, lint and wool.

(c) The provision of vessels delivering required quantities of sterile fluids, i.e. 'safesampling' fluid containers.

Both (b) and (c) are required for the cafeteria technique.

(d) Reduction of the multiplicity of cleansing lotions, antiseptics and therapeutic substances, applied to the wound. The average dressing trolley would be large enough for the proper practice of aseptic rituals, if it were not crowded with bottles and ointments. Most trolleys resemble a well-stocked cocktail bar.

(e) The provision of easily applied, flexible, occlusive dressings that do not require renewal. A man at work with a hand wound, which requires elaborate dressing, may return to the surgery every two hours for rebandaging, especially if he is working with fluids.

In collaboration with the hospital staff, industrial medical officers and nurses, we have attempted some of these technical improvements, but more investigation is required to define the most satisfactory materials for the initial cleansing of the wound, for use as an antibacterial dressing, and for the immobilization and cover of the wound.

As an example of the application of the principles enunciated above we may describe in some detail the reorganization of a temporary dressing station at the Birmingham Accident Hospital used for outpatients with established sepsis of wounds (Plans X and XI). Between 9 a.m. and 1 p.m. some 60-80 patients are seen and treated in the dressing station. In all cases the wound has to be inspected by a doctor; treatment may be simply a dressing, or may involve a preliminary soaking in a bath.

Plan X shows the original lay-out of the station. The general arrangements were as follows:

1. Patients entered by the door and sat on the bench (A) where they removed their dressings and put them in a large bin.

2. Patients were seen by the doctor (B) at a table near the middle of the room, and remote from a wash-basin and the light.

3. Having seen the doctor and had a temporary dressing, in the form of a piece of gauze, put on their wounds, the patients waited for their dressings on the other bench (C).

4. Dressings were done by two nurses working at a table near the window and wash-basin (E). They used the 'cafeteria' method. As will be seen from the plan, there was great congestion at this end of the room, in part because there were so many 


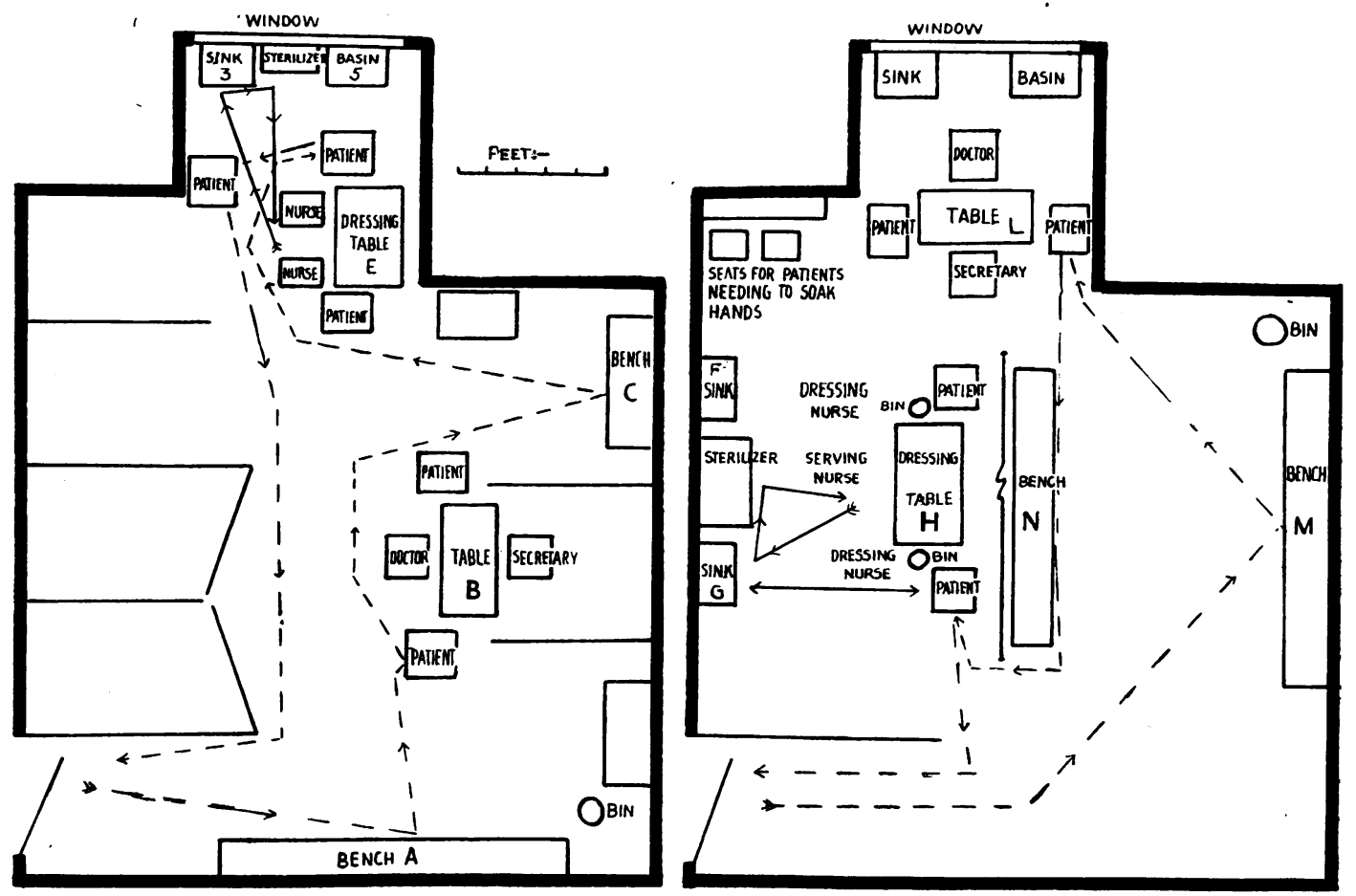

Plans X and XI.-Part of a temporary Out-Patient Department, Birmingham Accident Hospital, before and after reorganization. For key to letters, see text.

people congregated in a small area, and in part because the route taken by the patients crossed that followed by the nurses in taking instruments to the basin and sterilizer. The congestion was accentuated by the practice of having a runner to help with the bandaging, so that when the wound toilet was finished the patient had to move to another seat for bandaging.

The disadvantages of the plan may be summarized as, firstly, that the doctor saw the patients in a situation where the light was bad and whence, to get to a wash-basin, he had to go right through the collection of nurses and patients at the dressing table; and secondly, that the dressings were done, though near a wash-basin, in an area which made congestion inevitable. The result was that the nurses were unable to keep pace with the flow of patients, and a queue formed in the middle of the room.

The principles set out for the model factory surgery had to be modified somewhat in this case, since all patients were to be seen by a doctor before the wounds were dressed by the nurses. Nevertheless, the requirements were similar: all who have to handle wounds must be near a wash-basin; the dressings must be done near to the sterilizer; the paths followed by the patients and nurses must not cross; and inspection and treatment of wounds should be done in a good light.

The alterations made in the dressing station are shown in Plan XI. New wash-basins were inserted at (F) and (G) and the sterilizer was transferred to a table between them. This was then made the focal point for the dressers, a table being arranged at $(\mathrm{H})$, so that the nurses had access to basins and sterilizer, while the patients could easily reach their chairs. The team method of dressing, with a server, was introduced. The doctor's desk was transferred to the position (L) where he was under the window and near a wash-basin. Patients waiting to see him had plenty of space at (M), while those who had seen him and were waiting for dressings, could sit along the bench (N). A comparison of the routes followed by patients and staff on the Plans $\mathrm{X}$ and XI demonstrates the simplification achieved on paper, and this had been borne out in practice; the nurses are able to follow the safer dressing technique while taking less time over each patient.

\section{Summary and Conclusions}

Added infections during the treatment of wounds in a factory surgery produce manifest sepsis in a few cases and silent (clinically inapparent) infections in a much larger number of cases. Silent infection, which may be present in up to 40 per cent. of the wounds treated, not only delays healing but also creates a large reservoir of organisms capable of infecting clean wounds treated in the same surgery. Aseptic technique must therefore be designed to prevent cross-infection of wounds from those already septic or silently infected. Relative freedom from manifest sepsis is not a safe indication that the aseptic routine in use is fully efficient.

Aseptic techniques are often defective because the plan of the dressing station makes the practice of a completely safe routine unduly laborious. A revision of the technique usually implies a redesign of the dressing station. 
We have discussed the requirements of a dressing station which would allow the easy performance of a safe routine. The plan should be such that the dresser works conveniently close to the patient, the wash-basin and sterilizer, and the records desk, and that all unnecessary movements of the dresser are eliminated. Reorganization of a hospital dressing station along these lines enabled the staff to carry out a revised and safer dressing technique, with a decrease in the average time spent on each dressing.

Our detailed recommendations are, for the most part, trite, but we have so frequently observed the inconveniences which they are intended to remove that we have no hesitation in making them explicitly. We have also borne in mind that the factory surgery is used for other purposes beside wound dressing. The application of the principles of dressing station design need not interfere with -other functions of the factory surgery.

It should perhaps be emphasized that we consider the proper design of dressing stations to be a pre- liminary step in the control of wound infection. Only when added infection is minimized can the optimum methods of treatment of both clean and infected wounds be assessed with any confidence.

\section{Acknowledgements}

We are greatly indebted to the medical officers and nursing staffs of a large number of factory surgeries for co-operation in our survey and for much practical advice; and to our colleagues at the Birmingham Accident Hospital for their help.

\section{REFERENCES}

McKissock, W., Wright, Joyce, and Miles, A. A. (1941). Brit. med. J., 2, 375.

Medical Research Council. War Memo. No. 6. "The Prevention of Hospital Infection.' London, H.M.S.O. 1942.

Miles, A. A., Schwabacher, Herta, Cunliffe, A. C., Ross, J. P., Spooner, E. T. C., Pilcher, R. S., and Wright, Joyce (1940). Brit. med. J., 2, 855, 895.

Spooner, E. T. C. (1941). J. Hyg., Camb., 51, 320. 